\title{
Scattering of charmed baryons on nucleons
}

\author{
J. Haidenbauer ${ }^{1 \mathrm{a}}$ and G. Krein ${ }^{2 b}$ \\ 1 Institute for Advanced Simulation, Institut für Kernphysik, and Jülich Center for Hadron Physics, Forschungszentrum Jülich, \\ D-52425 Jülich, Germany \\ 2 Instituto de Física Teórica, Universidade Estadual Paulista \\ Rua Dr. Bento Teobaldo Ferraz, 271 - Bloco II, 01140-070, São Paulo, SP, Brazil
}

Received: date / Revised version: date

\begin{abstract}
Chiral effective field theory is utilized for extrapolating results on the $\Lambda_{c} N$ interaction, obtained in lattice QCD at unphysical (large) quark masses, to the physical point. The pion-mass dependence of the components that constitute the $\Lambda_{c} N$ potential up to next-to-leading order (pion-exchange diagrams and four-baryon contact terms) is fixed by information from lattice QCD simulations. No recourse to $\mathrm{SU}(3)$ or SU(4) flavor symmetry is made. It is found that the results of the HAL QCD Collaboration for quark masses corresponding to $m_{\pi}=410-570 \mathrm{MeV}$ imply a moderately attractive $\Lambda_{c} N$ interaction at $m_{\pi}=138 \mathrm{MeV}$ with scattering lengths of $a \approx-1 \mathrm{fm}$ for the ${ }^{1} S_{0}$ as well as the ${ }^{3} S_{1}$ partial waves. For such an interaction the existence of a charmed counterpart of the hypertriton seems unlikely but four- and/or five-baryons systems with a $\Lambda_{c}$ baryon could be indeed bound.
\end{abstract}

PACS. 14.20.Lq Charmed baryons $(|C|>0, \mathrm{~B}=0)$ - 12.39.Fe Chiral Lagrangians - 13.75.Ev Hyperonnucleon interactions -21.30 .Fe Forces in hadronic systems and effective interactions

\section{Introduction}

The dynamics of hadrons with different flavor degrees of freedom provides different windows for our understanding of the underlying theory of strong interaction, quantum chromodynamics (QCD). With regard to hadrons with charm, so far spectroscopy has been the most visible and definitely by far the most interesting branch of research. Indeed, the large number of structures observed in experiments at energies above the open charm production threshold provides a challenge for our standard (but obviously naive) picture that mesons are composed out of quark-antiquark pairs and baryons out of three quarks. See Refs. [1,2,3, 4] for recent overviews and discussions of these structures, commonly referred to as $\mathrm{X}, \mathrm{Y}$ and $\mathrm{Z}$ states.

Some proposals for experiments at sites such as JPARC [5] and FAIR 6, 7] aim at exploring also other aspects of charm physics. Specifically, so-called charm factories would allow the targeted production of charmed hadrons like the $D$-meson or the $\Lambda_{c}$ and $\Sigma_{c}$ hyperons and a study of their interaction with ordinary hadrons. The expectation of possible experiments in the not so far future has triggered a variety of theoretical investigations. In particular, it has led to a renewed interest in the interaction of the $\Lambda_{c}$ with nucleons and with nuclei over the last few years $8,9,10,11,12,13,14$. Those studies join

a j.haidenbauer@fz-juelich.de

b gkrein@ift.unesp.br the ranks of a long history of speculations about bound nuclear systems involving the $\Lambda_{c}$, the lightest charmed baryon [15, 16, 17, 18, 19, 20, 21, 22, — see also the recent reviews $[23,24$. Indeed, in most of the investigations so far, the $Y_{c} N$ interaction $\left(Y_{c}=\Lambda_{c}, \Sigma_{c}\right)$, derived within the meson-exchange framework $8,15,16,17,18$ or in the constituent quark model [12,25, turns out to be strongly attractive.

Interestingly, quite the opposite picture emerged from recent $(2+1)$-flavor lattice QCD (LQCD) simulations by the HAL QCD Collaboration [26,27,28]. Pertinent calculations, performed for unphysical quark masses corresponding to pion masses 11 of $m_{\pi}=410-700 \mathrm{MeV}$, suggest that the $\Lambda_{c} N$ and $\Sigma_{c} N$ interactions could be much less attractive than predicted by the phenomenological potentials mentioned above. While initial preliminary studies 26, 27 indicated an extremely weak $\Lambda_{c} N$ interaction, the recently published final LQCD results [28] rectify that conjecture and imply a somewhat stronger, though still only moderately attractive $\Lambda_{c} N$ interaction 2 .

In the present work we provide predictions for the $\Lambda_{c} N$ interaction at the physical point based on the LQCD

\footnotetext{
1 The Gell-Mann-Oakes-Renner relation states that the squared pion mass is proportional to the average light quark mass. Therefore, the notions "quark mass" and "pion mass" are used synonymously.

${ }^{2}$ We note that the method employed in these works and also other methods are presently under discussion in the LQCD community $29,30,31,32$.
} 
simulations by the HAL QCD Collaboration 28. The extrapolation of the LQCD results, available for $m_{\pi}=$ $410-700 \mathrm{MeV}$, to $m_{\pi}=138 \mathrm{MeV}$ is performed within the framework of chiral effective field theory (EFT) 33 , 34, 35. Thereby we follow a strategy that has been already employed by us in the past in the analysis of other LQCD results on baryon-baryon interactions in the strangeness sector, notably the $\Lambda \Lambda$ 36, $\Xi \Xi 37$, and $\Omega \Omega 38$ systems: At first chiral EFT is utilized to establish a $\Lambda_{c} N$ potential for baryon and meson masses that correspond to those in the lattice simulation. In particular, open parameters are determined by a fit to pertinent LQCD results (phase shifts, scattering lengths). Then the potential is extrapolated to the physical point. Thereby the pionmass dependence of the ingredients (pseudoscalar-meson exchange, four-baryon contact terms) is taken into account explicitly, and in line with chiral EFT.

The paper is structured as follows: In Sect. 2 we provide an outline of the employed formalism. Results for $\Lambda_{c} N$ phase shifts (for the ${ }^{1} S_{0}$ and ${ }^{3} S_{1}$ partial waves) are reported in Sect. 3, for pion masses corresponding to those in the LQCD simulation and for the physical value, $m_{\pi}=$ $138 \mathrm{MeV}$. The corresponding scattering lengths are evaluated too and turn out to be in the order of $a \approx-1 \mathrm{fm}$. Finally, consequences of our results for the existence of bound $\Lambda_{c}$ hypernuclei are discussed. The paper closes with a short summary.

\section{Formalism}

The $Y_{c} N$ interaction is constructed by using chiral EFT as guideline. Thereby we follow closely our application of this scheme to the $\Lambda N$ and $\Sigma N$ systems in Refs. 39, 40, where corresponding potentials have been obtained up to NLO in the Weinberg power counting 34,35. In this framework the potential is given in terms of pion exchanges and a series of contact interactions with an increasing number of derivatives. The latter represent the short-range part of the baryon-baryon force and are parameterized by lowenergy constants (LECs), that need to be fixed in a fit to data. Both classes of contributions depend on the quark mass (or, equivalently, the pion mass). At LO the only quark-mass dependence of the $Y_{c} N$ potential is through the pion mass that appears in the propagator of the pionexchange potential, cf. below. However, at NLO the contact terms as well as the pion coupling constants depend on the pion mass. For details, we refer to Refs. 41, 42, 43, 44, 45, 46, where the quark mass dependence of the $N N$ interaction has been investigated; see also Ref. [47.

Let us start by introducing the contact interaction that we employ. For the partial waves considered in the present study $\left({ }^{1} S_{0},{ }^{3} S_{1-}{ }^{3} D_{1}\right)$ it is given by

$$
\begin{aligned}
V\left({ }^{1} S_{0}\right)= & \tilde{C}_{{ }^{1} S_{0}}+\tilde{D}_{{ }^{1} S_{0}} m_{\pi}^{2} \\
& +\left(C_{{ }^{1} S_{0}}+D^{{ }^{1} S_{0}} m_{\pi}^{2}\right)\left(p^{2}+p^{2}\right), \\
V\left({ }^{3} S_{1}\right)= & \tilde{C}_{{ }^{3} S_{1}}+\tilde{D}_{{ }^{3} S_{1}} m_{\pi}^{2} \\
& +\left(C_{{ }^{3} S_{1}}+D^{3} S_{1} m_{\pi}^{2}\right)\left(p^{2}+p^{\prime 2}\right),
\end{aligned}
$$

$$
\begin{aligned}
& V\left({ }^{3} D_{1}-{ }^{3} S_{1}\right)=C_{\varepsilon_{1}} p^{\prime 2}, \\
& V\left({ }^{3} S_{1}-{ }^{3} D_{1}\right)=C_{\varepsilon_{1}} p^{2},
\end{aligned}
$$

with $p=|\mathbf{p}|$ and $p^{\prime}=\left|\mathbf{p}^{\prime}\right|$ being the initial and final center-of-mass (c.m.) momenta in the $\Lambda_{c} N$ or $\Sigma_{c} N$ systems. The quantities $\tilde{C}_{i}, \tilde{D}_{i}, C_{i}, D_{i}$ are the aforementioned LECs that need to be fixed by a fit to lattice data (phase shifts). The ansatz Eq. (1) is motivated by the corresponding expression in the standard Weinberg counting up to NLO [43, 47] but differs from it by the terms proportional to $m_{\pi}^{2}\left(p^{2}+p^{2}\right)$ which are nominally of higher order. Nevertheless, we include these terms because they allow us to obtain an optimal description of the LQCD results at $m_{\pi}=410 \mathrm{MeV}$ as well as at $570 \mathrm{MeV}$ and, thereby, enable us a better constrained extrapolation to lower pion masses. Contrary to our study of the $Y N$ interaction in Refs. 39, 40, here we do not impose SU(3) (or $\mathrm{SU}(4)$ ) flavor symmetry. In any case, given that there is no explicit information on the $\Sigma_{c} N$ channel from LQCD in Ref. 28] and the $\Lambda_{c} N$ interaction is determined as an effective single-channel potential, we treat the $\Lambda_{c} N$ interaction likewise as an effective single-channel problem.

The contribution of pion exchange to the $Y_{c} N$ potential is given by

$$
V_{B N \rightarrow B^{\prime} N}^{O P E}=-f_{B B^{\prime} \pi} f_{N N \pi} \frac{\left(\sigma_{1} \cdot \mathbf{q}\right)\left(\sigma_{2} \cdot \mathbf{q}\right)}{\mathbf{q}^{2}+m_{\pi}^{2}} \mathcal{I}_{B N \rightarrow B^{\prime} N}
$$

where $B, B^{\prime}$ stand for $\Lambda_{c}$ and/or $\Sigma_{c}, \mathbf{q}$ is the transferred momentum, $\mathbf{q}=\mathbf{p}^{\prime}-\mathbf{p}$, and $\mathcal{I}$ is a pertinent isospin factor 39. As already mentioned, we do not assume the validity of SU(4) flavor symmetry in the present study. This concerns also the coupling constants $f_{B B^{\prime} \pi}$. The $\Lambda_{c} \Sigma_{c} \pi$ coupling constant can be determined from the experimentally known $\Sigma_{c} \rightarrow \Lambda_{c} \pi$ decay rate, see Refs. [48, 49. With regard to the $\Sigma_{c} \Sigma_{c} \pi$ coupling constant we resort to LQCD results [50]. Besides their value at the physical point, we need also the $m_{\pi}$ dependence of the $\Lambda_{c} \Sigma_{c} \pi$ and $\Sigma_{c} \Sigma_{c} \pi$ coupling constants as well as the one for the $N N \pi$ vertex,

$$
f_{B B^{\prime} \pi}\left(m_{\pi}^{2}\right)=\frac{g_{A}^{B B^{\prime}}\left(m_{\pi}^{2}\right)}{2 F_{\pi}\left(m_{\pi}^{2}\right)}
$$

LQCD results for the $m_{\pi}^{2}$ dependence of the pion decay constant $F_{\pi}$ are readily available in the literature, e.g. in Ref. [51]. From that reference, one deduces the values $F_{\pi} \approx 112 \mathrm{MeV}$ at $m_{\pi}=410 \mathrm{MeV}, F_{\pi} \approx 129 \mathrm{MeV}$ at $m_{\pi}=570 \mathrm{MeV}$, and $F_{\pi} \approx 141 \mathrm{MeV}$ at $m_{\pi}=700 \mathrm{MeV}$. To obtain the latter value, a linear $m_{\pi}^{2}$ dependence of $F_{\pi}$ has been assumed, as suggested by Fig. 5 in Ref. [51. LQCD results for the $m_{\pi}^{2}$ dependence of the axial-vector strengths $g_{A}^{B B}$ can be found in Ref. [50] though only up to $m_{\pi}=500$ $\mathrm{MeV}$. For $g_{A}^{\Sigma_{c} \Sigma_{c}}$ a rather moderate increase with $m_{\pi}$ is suggested by LQCD [50]. $g_{A}^{N N}$ is found to be practically independent of $m_{\pi}$, though, unfortunately, the lattice results do not match well with the known value at the physical point. There is no information on the variation of the $\Lambda_{c} \Sigma_{c} \pi$ vertex with $m_{\pi}$. Because of these reasons we neglect the dependence of the $g_{A}$ 's on $m_{\pi}$ in our calculation and assume that $g_{A}^{B B^{\prime}} \equiv g_{A}^{B B^{\prime}}\left(m_{\pi}=138 \mathrm{MeV}\right)$. 
Specifically, we use $g_{A}^{N N}=1.27\left[52, g_{A}^{\Sigma_{c} \Sigma_{c}}=0.71\right.$ [50] and $g_{A}^{\Lambda_{c} \Sigma_{c}}=0.74$ 48, 49. Note that the by far strongest dependence of $f_{B B^{\prime} \pi}$ on $m_{\pi}^{2}$ comes via $F_{\pi}\left(m_{\pi}^{2}\right)$ [51] and this circumstance is adequately taken into account in our calculation.

Since $f_{\Lambda_{c} \Lambda_{c} \pi} \equiv 0$ under the assumption that isospin is conserved, there is no one-pion exchange contribution to the $\Lambda_{c} N \rightarrow \Lambda_{c} N$ potential. However, we include the coupling of $\Lambda_{c} N$ to $\Sigma_{c} N$ via pion exchange, which is known to play an important role in case of the $\Lambda N$ and $\Sigma N$ systems [40. The resulting effective two-pion exchange contribution to the $\Lambda_{c} N$ potential is generated by solving a coupled-channel Lippmann-Schwinger (LS) equation, see below. In this context let us note that the pertinent contribution arises anyway at NLO, even in a single-channel treatment 53 . In principle, at NLO there are further contributions from two-pion exchange 40. However, in the present study we omit those for simplicity reasons and assume that they are effectively absorbed into the contact terms. Furthermore, contributions from $\eta$ and $D$-meson exchanges that would arise under the assumption of $\mathrm{SU}(3)$ (or SU(4)) symmetry, are likewise delegated to the contact interactions.

As already mentioned above, in line with the analysis of the LQCD data in Ref. [28, we treat the $\Lambda_{c} N$ interaction as an effective single-channel problem. Possible effects from the interaction in the $\Sigma_{c} N \rightarrow \Sigma_{c} N$ channel are thereby absorbed into the LECs of the $\Lambda_{c} N$ potential. A further issue that we have ignored is the role of heavy quark spin symmetry [8,54. Indeed the $\Sigma_{c} N$ and $\Sigma_{c}^{*} N$ thresholds are just about $65 \mathrm{MeV}$ apart 52 so that the coupling between those systems could be important 8]. However, since already the $\Sigma_{c} N$ channel is not explicitly considered, there is no point in including $\Sigma_{c}^{*} N$ in our analysis. If there are any effects of it, these are likewise absorbed into the $\Lambda_{c} N$ LECs. Anyway, since $M_{\Sigma_{c}^{*}}-M_{\Lambda_{c}}=234 \mathrm{MeV}$, there should be less influence on the $\Lambda_{c} N$ amplitude. Indeed, because of the larger mass difference $M_{\Sigma_{c}}-M_{\Lambda_{c}} \approx 167 \mathrm{MeV}$, as compared to $M_{\Sigma}-$ $M_{\Lambda} \approx 78 \mathrm{MeV}$, one would expect that even the channel coupling to $\Sigma_{c} N$ plays a less important role for the charm sector than in the strangeness sector.

The reaction amplitudes are obtained from the solution of a coupled-channel LS equation for the interaction potentials, which is given in partial-wave projected form by

$$
\begin{aligned}
T_{\rho^{\prime} \rho}^{\nu^{\prime} \nu, J}\left(p^{\prime}, p ; \sqrt{s}\right)= & V_{\rho^{\prime} \rho}^{\nu^{\prime} \rho, J}\left(p^{\prime}, p\right) \\
& +\sum_{\rho^{\prime \prime}, \nu^{\prime \prime}} \int_{0}^{\infty} \frac{d p^{\prime \prime} p^{\prime \prime 2}}{(2 \pi)^{3}} V_{\rho^{\prime} \rho^{\prime \prime}}^{\nu^{\prime \prime}, J}\left(p^{\prime}, p^{\prime \prime}\right) \\
& \times \frac{2 \mu_{\rho^{\prime \prime}}}{p_{\rho}^{2}-p^{\prime \prime 2}+i \eta} T_{\rho^{\prime \prime} \rho}^{\nu^{\prime \prime} \nu, J}\left(p^{\prime \prime}, p ; \sqrt{s}\right) .(4)
\end{aligned}
$$

Here, the label $\rho$ indicates the particle channels and the label $\nu$ the partial wave. $\mu_{\rho}$ is the pertinent reduced mass. The on-shell momentum in the intermediate state, $p_{\rho}$, is defined by $\sqrt{s}=\sqrt{m_{B_{1, \rho}}^{2}+p_{\rho}^{2}}+\sqrt{m_{B_{2, \rho}}^{2}+p_{\rho}^{2}}$. Following the practice of the HAL QCD Collaboration 28, phase shifts will be given as functions of the kinetic energy in the $\Lambda_{c} N$ c.m. frame, $E=\sqrt{s}-M_{\Lambda_{c}}-M_{N}$.

For baryon-baryon potentials constructed along the lines of chiral EFT (cf. Eqs. (1) and (2)) a regularization is required when solving the LS equation (44) 33, 35. Ideally, the resulting reaction amplitude $T$ should be completely independent of the employed regularization scheme. In practise, there is still a dispute about how regularization should be done in the application of chiral EFT to $N N$ scattering (and accordingly in the $Y N$ or $Y_{c} N$ case) to satisfy all formal aspects from a field theory point of view and there is no generally accepted scheme, cf. Refs. 33, 35 and references therein. A commonly accepted procedure is the introduction of a cutoff into the Lippmann-Schwinger equation or (equivalently) to the potential. The controversial issue is, however, how one should then proceed in detail in order to achieve the desired cutoff independence of the results see, e.g. [55, 56, 57, 58, 59,60,61.

In the present work we refrain from elaborating on this still open question. Rather we follow strictly the procedure used in the application of chiral EFT to $N N$ scattering by Epelbaum and collaborators 56] and in our $Y N$ studies [40], where the potentials in the LS equation are cut off in momentum space by multiplication with a regulator function, $f\left(p^{\prime}, p\right)=\exp \left[-\left(p^{\prime 4}+p^{4}\right) / \Lambda^{4}\right]$, so that the highmomentum components of the baryon and pseudoscalar meson fields are removed. The cutoff parameter $\Lambda$ in the regulator is typically in the order of $\Lambda \approx 500 \mathrm{MeV} 34$, 35 . It is kept finite in the calculation. Approximate cutoff independence is achieved by going to higher orders in the perturbative expansion of the potential where the sucessively arising contact terms allow one to absorb/remove the cutoff dependence more and more efficiently [60. The actual values we employ for the cut-off are $\Lambda=500-600$ $\mathrm{MeV}$, in line with the range that yielded optimal and stable results in our NLO study of the $\Lambda N$ and $\Sigma N$ interactions 40 . The variations of the results with the cutoff, i.e. the residual cutoff dependence, reflect uncertainties that will be indicated by bands in the plots we show in the next section.

In the analysis of the LQCD simulations we follow closely the strategy of our previous works in Refs. 36. 37,38: (i) The LECs, i.e. the only free parameters in the potential, are determined by a fit to LQCD results (phase shifts) employing the inherent baryon and meson masses of the lattice simulation; (ii) Results at the physical point are obtained via a calculation in which the pertinent physical masses of the mesons are substituted in the evaluation of the potential and those of the baryons in the baryonbaryon propagators appearing in the LS equation. The baryon masses corresponding to the LQCD simulations at $m_{\pi}=410$ and $570 \mathrm{MeV}$ are taken from Ref. 28. For the calculation at the physical point we use the masses from the PDG [52], i.e. $M_{\Lambda_{c}}=2286.5 \mathrm{MeV}, M_{\Sigma_{c}}=2455 \mathrm{MeV}$.

Alternative ways to implement the quark mass dependence of the nuclear forces can be found in Refs. [45, 62 . 
Table 1. Low-energy constants employed in the present work. $\tilde{C}_{{ }^{S_{0}}} \equiv \tilde{C}_{{ }_{S_{0}}}+\tilde{D}_{{ }_{S_{0}}} m_{\pi}^{2}$ at the specified pion mass, etc., see Eq. (1). The values for $\tilde{C}$ are in $10^{4} \mathrm{GeV}^{-2}$, those for $C$ in $10^{4} \mathrm{GeV}^{-4}$.

\begin{tabular}{||l||c|c||c|c||c|c||}
\hline \hline \multicolumn{1}{||c||}{} & \multicolumn{2}{c||}{$570 \mathrm{MeV}$} & \multicolumn{2}{c||}{$410 \mathrm{MeV}$} & \multicolumn{2}{c||}{$138 \mathrm{MeV}$} \\
\hline \hline$\Lambda=500 \mathrm{MeV}$ & $\tilde{C}$ & $C$ & $\tilde{C}$ & $C$ & $\tilde{C}$ & $C$ \\
\hline${ }^{1} S_{0}$ & -0.008576 & -0.006105 & -0.01758 & -0.01362 & -0.02615 & -0.02077 \\
${ }^{3} S_{1}$ & 0.03917 & 0.04569 & 0.1409 & 0.1270 & 0.2377 & 0.2043 \\
${ }^{3} D-{ }^{3} S_{1}$ & & -0.1136 & & -0.1136 & & -0.1136 \\
\hline \hline$\Lambda=600 \mathrm{MeV}$ & $\tilde{C}$ & $C$ & $\tilde{C}$ & $C$ & $\tilde{C}$ & $C$ \\
\hline${ }^{1} S_{0}$ & -0.008026 & 0.001564 & -0.01393 & -0.002252 & -0.01954 & -0.005879 \\
${ }^{3} S_{1}$ & 0.04304 & 0.07553 & 0.1619 & 0.1688 & 0.2749 & 0.2574 \\
${ }^{3} D-{ }^{3} S_{1}$ & & -0.1344 & & -0.1344 & & -0.1344 \\
\hline \hline
\end{tabular}

\section{Results}

LQCD results for phase shifts are available for the $\Lambda_{c} N$ ${ }^{1} S_{0}$ and ${ }^{3} S_{1}$ partial waves for $m_{\pi}=410,570,700 \mathrm{MeV}$ 28. We determine the LECs of the contact interaction, cf. Eq. (11), by a fit to the lattice data at the two lower pion masses. Specifically, the pion-mass dependence exhibited by the LQCD simulation is exploited to determine the LECs $\tilde{D}_{i}$ and $D_{i}$ that encode the pion-mass dependence of the contact interaction. The fits are done to the phase shifts, generated from the parameterized version of the $\Lambda_{c} N$ potentials provided in Ref. 28, for energies up to $30 \mathrm{MeV}$. Alternative fits taking into account the HAL QCD results up to $50 \mathrm{MeV}$ were performed too. In this context it should be noted that applications of chiral EFT to $N N$ scattering and specifically to the ${ }^{1} S_{0} n p$ state reveal that NLO interactions are expected to provide quantitative results up to roughly $50 \mathrm{MeV}$ [34,35. The actual values of the LECs at the fitted pion masses and at the physical value are summarized in Table 1.

Results for the ${ }^{1} S_{0}$ partial wave are presented in Fig. 1 The phase shifts for pion masses 570, 410 and $138 \mathrm{MeV}$ are shown on the left side while the dependence of the scattering length on the pion mass is depicted on the right side. The bands represent the dependence of the results on variations of the cutoff $\Lambda$. One can see that the lattice results at $m_{\pi}=410 \mathrm{MeV}$ are reproduced quantitatively by our potential up to c.m. kinetic energies of around $40 \mathrm{MeV}$, as expected for an NLO interaction, while those at $570 \mathrm{MeV}$ are remarkably well described over the whole energy range shown. In both cases the cutoff dependence is negligibly small at low energies so that the bands are hardly visible. The phase shift obtained from our interaction when extrapolated to the physical point (red bands) do exhibit a noticeable but still rather moderate cutoff dependence. A maximum of around 20 degrees of the ${ }^{1} S_{0}$ phase shift is predicted in this case. The pion-mass dependence of the ${ }^{1} S_{0}$ scattering length, shown on the right-hand side of Fig. [1] is fairly smooth and almost linear in $m_{\pi}$. Only close to the physical point a somewhat stronger $m_{\pi}$ de- pendence is visible. The value predicted at $138 \mathrm{MeV}$ is $a=-0.85 \cdots-1.0 \mathrm{fm}$.

Results for the ${ }^{3} S_{1}$ partial wave are presented in Fig. 2 Again phase shifts as well as the pion-mass dependence of the scattering length are shown. Those are very similar to the ones of the ${ }^{1} S_{0}$ state, even on a quantitative level. Indeed, it was already noted by the HAL QCD Collaboration that the corresponding $\left({ }^{1} S_{0}\right.$ and $\left.{ }^{3} S_{1}\right)$ potentials they extracted are almost identical - at 410 as well as at $570 \mathrm{MeV}$ [28], and we see that this characteristic feature persists even in our extrapolation to the physical point. This is certainly a remarkable feature in view of the fact that in case of the ${ }^{3} S_{1}$ partial wave there is a coupling to the ${ }^{3} D_{1}$ induced by the tensor force, and this coupling is taken into account in our analysis.

For completeness we have summarized the results for the ${ }^{1} S_{0}$ and ${ }^{3} S_{1}$ scattering lengths, $a_{s}$ and $a_{t}$, in Table 2 . From those numbers one can see that the variation in the extrapolated values due to the employed regularization scheme is in the order of $0.2 \mathrm{fm}$. Additional fits to the phase shifts where the considered energy range was extended up to $50 \mathrm{MeV}$ led to changes in the scattering lengths at the physical point of around $0.1 \mathrm{fm}$, with a clear tendency to smaller values. Further exploratory fits carried out by us indicate that variations in the scattering length of $\pm 0.05 \mathrm{fm}$ at $m_{\pi}=410 \mathrm{MeV}$ amount to differences of about $\pm 0.10 \mathrm{fm}$ at $m_{\pi}=138 \mathrm{MeV}$. Combining these observations with the uncertainty of $\pm 0.2 \mathrm{fm}$ given by the HAL QCD Collaboration for their result at $m_{\pi}=410 \mathrm{MeV}$ suggests that the scattering lengths at the physical point could be about $0.3 \mathrm{fm}$ larger, i.e. as large as $-1.3 \mathrm{fm}$. Finally, for estimating possible effects from the $\Sigma_{c} N$ channel we performed calculations with the pion-exchange contribution (2) to the $\Sigma_{c} N$ potential included. Adding its contribution within a coupled-channel framework (4) has a negligible influence on the results at $m_{\pi}=410 \mathrm{MeV}$ and $570 \mathrm{MeV}$, but leads to noticeable variations in the predictions for $m_{\pi}=138 \mathrm{MeV}$ that amount to roughly $0.3 \mathrm{fm}$ in the scattering lengths. Of course, a complete calculation has to include also a contact term in the $\Sigma_{c} N$ channel, which could facilitate a 

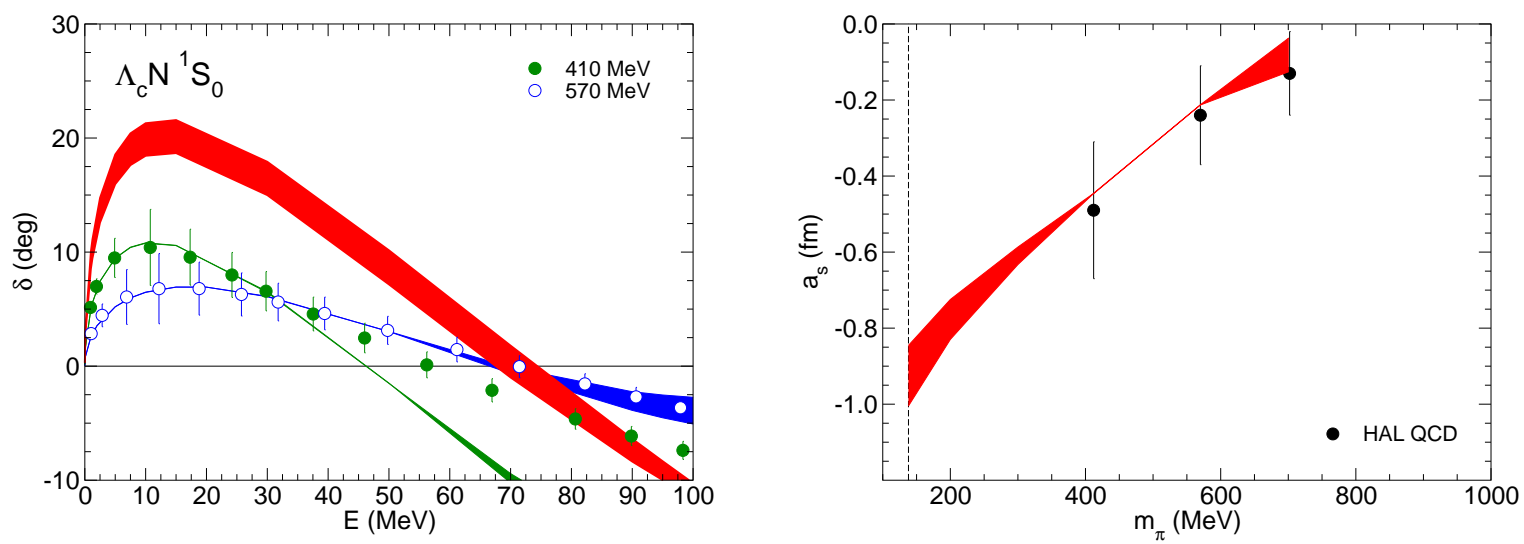

Fig. 1. Results for the $\Lambda_{c} N{ }^{1} S_{0}$ partial wave. Left: Phase shifts of the HAL QCD Collaboration 28 at $m_{\pi}=570 \mathrm{MeV}$ (blue open circles) and $410 \mathrm{MeV}$ (green filled circles), as a function of the c.m. kinetic energy, together with our fits (lines/bands). The broader (red) band is the prediction for $m_{\pi}=138 \mathrm{MeV}$. Right: Dependence of the ${ }^{1} S_{0}$ scattering length on the pion mass. The bands represent the cutoff variation $\Lambda=500-600 \mathrm{MeV}$, see text.
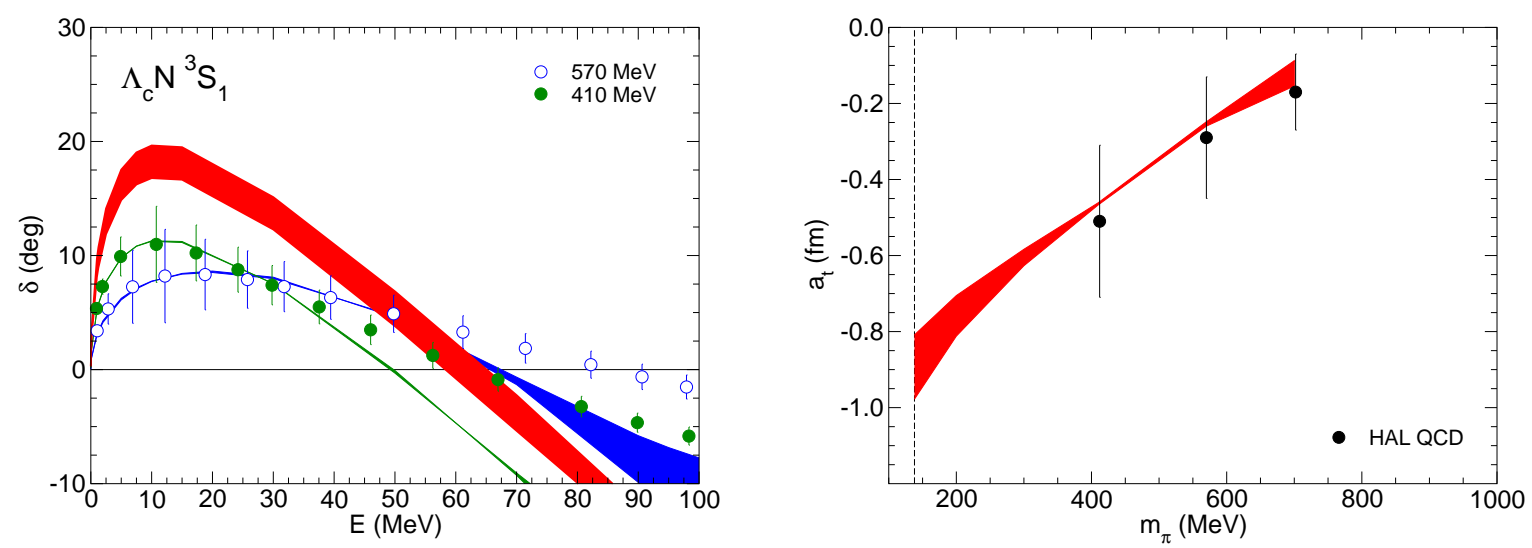

Fig. 2. Results for the $\Lambda_{c} N{ }^{3} S_{1}$ partial wave. Left: Phase shifts of the HAL QCD Collaboration 28 at $m_{\pi}=570 \mathrm{MeV}$ (blue open circles) and $410 \mathrm{MeV}$ (green filled circles), as a function of the c.m. kinetic energy, together with our fits (lines/bands). The broader (red) band is the prediction for $m_{\pi}=138 \mathrm{MeV}$. Right: Dependence of the ${ }^{3} S_{1}$ scattering length on the pion mass. The bands represent the cutoff variation $\Lambda=500-600 \mathrm{MeV}$, see text.

reduction of the effect. Obviously, additional information on the $\Sigma_{c} N$ phase shifts, as had been provided in the preliminary study of the HAL QCD Collaboration [27, and promised in Ref. 28 for the future, would be helpful for reducing the uncertainty in the extrapolation. In any case, a more quantitative estimate of the overall uncertainty should be attempted, once lattice data with better statistics are available and/or results for pion masses closer to the physical point.

Clearly, and in line with the LQCD results for larger pion masses, we do not get any $\Lambda_{c} N$ bound states. However, what are the consequences of the results presented in the preceding paragraphs for the possible existence of bound $\Lambda_{c}$ nuclei? Let us look at the strangeness sector and, specifically, at the lightest $\Lambda$ nucleus that is experimen- tally observed, namely the hypertriton ${ }^{3}{ }_{\Lambda} \mathrm{H}$. The experimental value for the ${ }^{3} \mathrm{H}$ binding energy is $(-2.354 \pm 0.050)$ $\mathrm{MeV}$, which implies a separation energy for the $\Lambda$ of only $(0.13 \pm 0.05) \mathrm{MeV}$. Faddeev calculations of the coupled $\Lambda N N-\Sigma N N$ three-body systems for realistic $Y N$ potentials have been reported in Refs. 63, 64,65. Those suggest that, for $Y N$ interactions which provide sufficient attraction so that the hypertriton is bound, the $\Lambda N$ scattering lengths are in the order of -2.9 to $-2.6 \mathrm{fm}$ for the ${ }^{1} S_{0}$ state and -1.5 to -1.7 fm for ${ }^{3} S_{1}$.

The scattering lengths for the $\Lambda_{c} N$ system at the physical point, deduced from the LQCD simulations of the HAL QCD Collaboration, are -0.8 to -1.0 fm for the ${ }^{1} S_{0}$ as well as the ${ }^{3} S_{1}$ states. Thus, in the case of the ${ }^{1} S_{0}$ partial wave the value is considerably smaller than its counterpart 
Table 2. $\Lambda_{c} N$ scattering lengths in the ${ }^{1} S_{0}\left(a_{s}\right)$ and ${ }^{3} S_{1}\left(a_{t}\right)$ partial waves (in fm) for different pion masses. Predictions for the corresponding effective range parameters $r$ (in $\mathrm{fm}$ ) at the physical point are likewise given.

\begin{tabular}{c|l||c|c|c|c}
\hline \hline \multicolumn{1}{c||}{$m_{\pi}$} & $700 \mathrm{MeV}$ & $570 \mathrm{MeV}$ & $410 \mathrm{MeV}$ & $138 \mathrm{MeV}$ \\
\hline \hline$a_{s}$ & HAL QCD [28] & $-0.13 \pm 0.11$ & $-0.24 \pm 0.13$ & $-0.49 \pm 0.18$ & - \\
& our results & $-0.04 \cdots-0.13$ & -0.21 & -0.45 & $-0.85 \cdots-1.00$ \\
$r_{s}$ & & & & & $2.88 \cdots 2.61$ \\
\hline$a_{t}$ & HAL QCD [28] & $-0.17 \pm 0.10$ & $-0.29 \pm 0.16$ & $-0.51 \pm 0.20$ & - \\
& our results & $-0.09 \cdots-0.15$ & $-0.25 \cdots-0.26$ & $-0.46 \cdots-0.47$ & $-0.81 \cdots-0.98$ \\
$r_{t}$ & & & & $3.50 \cdots 3.15$ \\
\hline \hline
\end{tabular}

in the strangeness sector while for the ${ }^{3} S_{1}$ channel the difference is less dramatic. Since the average $\Lambda N\left(\right.$ or $\left.\Lambda_{c} N\right)$ potential that is relevant for the hypertriton is dominated by the spin-singlet channel, i.e. $V_{\Lambda N}\left(V_{\Lambda_{c} N}\right) \propto \frac{3}{4} V_{s}+\frac{1}{4} V_{t}$, see Ref. [66] for details, it seems rather unlikely that an only moderately attractive singlet $\Lambda_{c} N$ interaction can support the existence of a ${ }_{1}^{3} \mathrm{H}$ bound state. Even effects due to a reduction of the kinetic energy associated with the $\Lambda_{c}$ induced by the larger mass of the $\Lambda_{c}$ as compared to the $\Lambda$, emphasized in several works in the past [11, 15, 18, cannot compensate for this large difference in the strength. Actually, in Ref. 18 an estimate for the binding energy of charmed three- and four-body systems is provided based on an exact solution of corresponding scattering equations. Interestingly, Model 4 considered in that work yields scattering lengths very similar to the ones of our analysis, namely $a_{s}=-1.075 \mathrm{fm}$ and $a_{t}=-0.828 \mathrm{fm}$ (cf. Table I in that work). For this interaction no bound state is found for the ${ }_{\Lambda_{c}^{3}}^{3} \mathrm{H}$ nucleus. However, the 4-body systems ${ }_{\Lambda_{c}^{4}}^{4} \mathrm{He}$ and ${ }_{\Lambda_{c}^{4}}^{4} \mathrm{Li}$ could be already bound, though possibly only weakly, even when considering the additional repulsive effect from the Coulomb force due to the charge of the $\Lambda_{c}^{+}$ as discussed in Ref. 18.

With regard to recent few-body calculations, the $Y_{c} N$ interaction employed in Ref. 12 is strongly attractive and leads already to $\Lambda_{c} N$ bound states. The binding energies for $\Lambda_{c} N N$ are then in the order of $20 \mathrm{MeV}$. Those results do not allow us to draw any conclusions about what would happen for significantly less attractive $Y_{c} N$ interactions like the one we deduce from the analysis of the LQCD simulations by the HAL QCD Collaboration. In case of the three-body calculation presented in Ref. [11, a charmed hypertriton with $J=3 / 2$ (and total isospin $I=0$ ) is predicted. For that state the $\Lambda_{c} N$ spin-triplet interaction is dominant 67]. However, since there is no information on the scattering length and effective range parameters of the $\Lambda_{c} N$ potential employed in Ref. [11, a proper assessment of that result is difficult.

\section{Summary}

In the present work, we have used the framework of chiral effective field theory to extrapolate lattice QCD results for the $\Lambda_{c} N$ interaction at $m_{\pi}=410-570 \mathrm{MeV}$ by the HAL QCD Collaboration [28] to the physical point. Thereby, we have followed a strategy employed previously in the strangeness sector $36,37,38$. However, contrary to the calculations performed in those works, no recourse to $\mathrm{SU}(3)$ or $\mathrm{SU}(4)$ flavor symmetry has been made in the present study. Furthermore, in the present work the pionmass dependence of all components that constitute the $\Lambda_{c} N$ potential up to next-to-leading order (pion-exchange diagrams and four-baryon contact terms) are taken into account. Information from lattice QCD simulations is utilized to implement these features.

Our analysis of the HAL QCD results points to a moderately attractive $\Lambda_{c} N$ interaction at the physical point with scattering lengths of $a \approx-1 \mathrm{fm}$ for the ${ }^{1} S_{0}$ as well as for the ${ }^{3} S_{1}$ partial waves. Such an interaction leads to the possibility of bound four- and/or five-baryons systems with a $\Lambda_{c}$ baryon and presumably of heavier $\Lambda_{c}$ hypernuclei. On the other hand, two-body $\left(\Lambda_{c} N\right)$ bound states as advocated in some recent investigations based on phenomenological potentials [8, 12 can be definitely excluded, even if one considers uncertainties in the extrapolation of the lattice results. Also, the existence of a hypertriton-like $\Lambda_{c} N N$ three-body bound state (with $J=1 / 2$ ) seems rather unlikely if one takes past investigations [18] as benchmark.

\section{Acknowledgments}

Work partially supported by Conselho Nacional de Desenvolvimento Científico e Tecnológico (CNPq), Grant No. 305894/2009-9 (G.K.) and Grant No. 464898/2014-5 (G.K.) (INCT Física Nuclear e Applicações), Fundação de Amparo à Pesquisa do Estado de São Paulo (FAPESP), Grant No. 2013/01907-0 (G.K.)

\section{References}

1. F.-K. Guo, C. Hanhart, U.-G. Meißner, Q. Wang, Q. Zhao and B.-S. Zou, Rev. Mod. Phys. 90 (2018) 015004.

2. R. F. Lebed, R. E. Mitchell and E. S. Swanson Prog. Part. Nucl. Phys. 93 (2017) 143.

3. H.-X. Chen, W. Chen, X. Liu, Y.-R. Liu and S.-L. Zhu, Rept. Prog. Phys. 80 (2017) 076201.

4. A. Esposito, A. Pilloni and A. D. Polosa, Phys. Rept. 668 (2016) 1. 
5. H. Noumi et al., Proposal P50, J-PARC PAC-16, 2013 http://j-parc.jp/researcher/Hadron/en/pac_1301/pdf/ P50_2012-19.pdf

6. B. Friman, C. Hohne, J. Knoll, S. Leupold, J. Randrup, R. Rapp and P. Senger, Lect. Notes Phys. 814 (2011) 1.

7. U. Wiedner, Prog. Part. Nucl. Phys. 66 (2011) 477.

8. Y. R. Liu and M. Oka, Phys. Rev. D 85 (2012) 014015.

9. H. Huang, J. Ping and F. Wang, Phys. Rev. C 87 (2013) 034002.

10. A. Gal, H. Garcilazo, A. Valcarce and T. FernándezCaramés, Phys. Rev. D 90 (2014) 014019.

11. H. Garcilazo, A. Valcarce and T. F. Caramés, Phys. Rev. C 92 (2015) 024006.

12. S. Maeda, M. Oka, A. Yokota, E. Hiyama and Y. R. Liu, PTEP 2016 (2016) 023D02.

13. R. Shyam and K. Tsushima, Phys. Lett. B 770 (2017) 236.

14. K. Ohtani, K. J. Araki and M. Oka, Phys. Rev. C 96 (2017) 055208 .

15. C. B. Dover and S. H. Kahana, Phys. Rev. Lett. 39 (1977) 1506.

16. H. Bando and M. Bando, Phys. Lett. B 109 (1982) 164.

17. H. Bando and S. Nagata, Prog. Theor. Phys. 69 (1983) 557.

18. B. F. Gibson, G. Bhamathi, C. B. Dover and D. R. Lehman, Phys. Rev. C 27 (1983) 2085.

19. S. A. Bunyatov, V. V. Lyukov, N. I. Starkov and V. A. Isarev, Sov. J. Part. Nucl. 23 (1992) 253.

20. K. Tsushima and F.C. Khanna Phys. Rev. C 67 (2003) 015211

21. K. Tsushima and F.C. Khanna, J. Phys. G 30 (2004) 1765.

22. V. B. Kopeliovich and A. M. Shunderuk, Eur. Phys. J. A 33 (2007) 277.

23. A. Hosaka, T. Hyodo, K. Sudoh, Y. Yamaguchi and S. Yasui, Prog. Part. Nucl. Phys. 96 (2017) 88.

24. G. Krein, A. W. Thomas and K. Tsushima, Prog. Part. Nucl. Phys. 100 (2018) 161.

25. F. Frömel, B. Juliá-Díaz and D. O. Riska, Nucl. Phys. A 750 (2005) 337

26. T. Miyamoto [HAL QCD Collaboration], PoS LATTICE 2015 (2016) 090.

27. T. Miyamoto [HAL QCD Collaboration], PoS LATTICE 2016 (2017) 117.

28. T. Miyamoto et al., Nucl. Phys. A 971 (2018) 113.

29. S. Aoki, T. Doi and T. Iritani, EPJ Web Conf. 175 (2018) 05006 .

30. T. Yamazaki and Y. Kuramashi, Phys. Rev. D 96 (2017) 114511.

31. Z. Davoudi, EPJ Web Conf. 175 (2018) 01022.

32. S. Aoki, T. Doi, T. Hatsuda and N. Ishii, arXiv:1711.09344 [hep-lat].

33. E. Epelbaum, Prog. Part. Nucl. Phys. 57 (2006) 654.

34. E. Epelbaum, H. W. Hammer and U.-G. Meißner, Rev. Mod. Phys. 81 (2009) 1773.

35. R. Machleidt and D. R. Entem, Phys. Rep. 503 (2011) 1.

36. J. Haidenbauer and U.-G. Meißner, Phys. Lett. B 706 (2011) 100

37. J. Haidenbauer and U.-G. Meißner, Nucl. Phys. A 881 (2012) 44.

38. J. Haidenbauer, S. Petschauer, N. Kaiser, U.-G. Meißner and W. Weise, Eur. Phys. J. C 77 (2017) 760.

39. H. Polinder, J. Haidenbauer, and U.-G. Meißner, Nucl. Phys. A 779 (2006) 244.
40. J. Haidenbauer, S. Petschauer, N. Kaiser, U.-G. Meißner, A. Nogga and W. Weise, Nucl. Phys. A 915 (2013) 24.

41. S. R. Beane, M. J. Savage, Nucl. Phys. A 713 (2003) 148. 42. S. R. Beane, M. J. Savage, Nucl. Phys. A 717 (2003) 91.

43. E. Epelbaum, U.-G. Meißner, W. Glöckle, Nucl. Phys. A 714 (2002) 535.

44. E. Epelbaum, U.-G. Meißner, W. Glöckle, arXiv:nucl-th/0208040.

45. V. Baru, E. Epelbaum, A. A. Filin and J. Gegelia, Phys. Rev. C 92 (2015) 014001.

46. V. Baru, E. Epelbaum and A. A. Filin, Phys. Rev. C 94 (2016) 014001.

47. S. Petschauer and N. Kaiser, Nucl. Phys. A 916 (2013) 1.

48. C. Albertus, E. Hernandez, J. Nieves and J. M. Verde-

Velasco, Phys. Rev. D 72 (2005) 094022.

49. K. U. Can, G. Erkol, M. Oka and T. T. Takahashi, Phys. Lett. B 768 (2017) 309.

50. C. Alexandrou, K. Hadjiyiannakou and C. Kallidonis, Phys. Rev. D 94 (2016) 034502.

51. S. Dürr et al. [Budapest-Marseille-Wuppertal Collaboration], Phys. Rev. D 90 (2014) 114504.

52. C. Patrignani et al. (Particle Data Group), Chin. Phys. C 40 (2016) 100001.

53. S. R. Beane, P. F. Bedaque, A. Parreño and M. J. Savage, Nucl. Phys. A 747 (2005) 55.

54. J.-X. Lu, L.-S. Geng and M. Pavón Valderrama, arXiv:1706.02588 [hep-ph].

55. G. P. Lepage, nucl-th/9706029.

56. E. Epelbaum, W. Glöckle, U.-G. Meißner, Nucl. Phys. A 747 (2005) 362.

57. A. Nogga, R. G. E. Timmermans and U. van Kolck, Phys. Rev. C 72 (2005) 054006.

58. E. Marji, A. Canul, Q. MacPherson, R. Winzer, C. Zeoli, D. R. Entem and R. Machleidt, Phys. Rev. C 88 (2013) 054002 .

59. D. R. Phillips, PoS CD 12 (2013) 013.

60. E. Epelbaum, H. Krebs and U.-G. Meißner, Eur. Phys. J. A 51 (2015) 53.

61. E. Epelbaum, J. Gegelia and U.-G. Meißner, Nucl. Phys. B 925 (2017) 161.

62. J. C. Berengut, E. Epelbaum, V. V. Flambaum, C. Hanhart, U.-G. Meißner, J. Nebreda and J. R. Pelaez, Phys. Rev. D 87 (2013) 085018.

63. A. Nogga, H. Kamada and W. Glöckle, Phys. Rev. Lett. 88 (2002) 172501.

64. J. Haidenbauer, U.-G. Meißner, A. Nogga and H. Polinder, Lect. Notes Phys. 724 (2007) 113.

65. A. Nogga, Nucl. Phys. A 914 (2013) 140.

66. B. F. Gibson, I. R. Afnan, J. A. Carlson and D. R. Lehman, Prog. Theor. Phys. Suppl. 117 (1994) 339.

67. K. Miyagawa, H. Kamada, W. Glöckle and V. Stoks, Phys. Rev. C 51 (1995) 2905. 